\title{
THE MECHANICAL PROPERTIES OF THE LUNGS IN EMPHYSEMA ${ }^{1}$
}

\author{
By J. MEAD, I. LINDGREN, AND E. A. GAENSLER
} (From the Department of Physiology, Harvard School of Public Health and the Thorndike
Memorial Laboratory, Second and Fourth Medical Services (Harvard), Boston

City Hospital, Boston, Mass.)

(Submitted for publication January 3, 1955; accepted March 9, 1955)

In pulmonary emphysema normal respiratory mechanisms become distorted almost beyond recognition, and familiar landmarks such as "dead space," "alveolar gas" and "effective ventilation" virtually disappear. At the same time, these very distortions have served as a stimulus for studies which have led to a greater understanding of the problems of distribution of ventilation, and of blood flow within the lungs.

In attempting to study the mechanics of breathing in emphysema, the authors have also found that concepts based on the behavior of normal lungs are inadequate to account for their observations. This has led to a consideration of the influence of local mechanical factors on the over-all mechanical properties of the lungs. Since these considerations are also pertinent to problems of the distribution of gas flow within the lungs, and possibly also to the pathogenesis of emphysema, they will be emphasized in this report. The details of the theoretical treatment will appear elsewhere.

\section{METHODS}

Ten patients with advanced pulmonary emphysema and ten normal individuals have been studied. Details of the methods have been reported elsewhere (1).

\section{Methods of measurement}

Rates of flow were recorded at the mouth with a Silverman pneumotachograph screen connected to a manometer. Volume changes were measured by electrical integration of the rate of flow signal coming from the pneumotachograph manometer. This method of recording rates of gas flow and volume changes has the advantages of excellent response characteristics and of imposing a negligible external resistance. It has the disadvantage that volume changes occurring over a period greater than fifteen seconds cannot be recorded accurately because of the limitations imposed by the integrator. For such slow volume changes a recording spirometer was used.

${ }^{1}$ Aided by a research grant from the National Tuberculosis Association.
As a measure of transpulmonary pressures the pressure difference between the mouth and a point within the lower third of the esophagus was recorded. The intra-esophageal pressure was transmitted to one side of a differential manometer by means of an air-filled polyethylene tube $64 \mathrm{~cm}$. in length, $1 \mathrm{~mm}$. in internal diameter, with eight to ten spirally arranged holes in the catheter wall at the distal end. A thin-walled rubber balloon with a volume of $1.5 \mathrm{ml}$. undistended and $3.2 \mathrm{~cm}$. in length was sealed to the catheter and enclosed the openings. The other side of the manometer membrane was connected to the mouthpiece chamber by pressure tubing. The catheter was passed through the nose and was readily swallowed. The air volume of the balloon during recording was 0.5 to $1.0 \mathrm{ml}$. The flow rates, volumes and pressures were recorded simultaneously on a direct-writing oscillograph.

The pressure difference existing at any instant between the mouth and the surface of the lungs depends on the elastic recoil of the lungs and the flow-resistance of the airways and lung tissue. The elastic component of pressure increases as the lungs are inflated, and the relationship of lung volume change to the corresponding elastic pressure change serves as a measure of the lungs' elastic properties. The flow-resistive component of pressure depends on the rate at which the lungs are changing volume, and the relationship of this component to the simultaneous rate of volume change of the lungs serves as a measure of the lung and airway flow-resistive properties.

In analyzing the simultaneous recordings of volumes, rates of flow, and pressures in order to obtain information about the mechanical properties of the lungs, the principal step is the separation of the total pressure into its elastic and flow-resistive components. This separation may be obtained by graphic means from measurements of the Poly-Viso tracing. 2 This tedious procedure may be avoided, however, by using a cathode-ray oscilloscope to plot the variables.

Two types of patterns were produced on the oscilloscope face. In one (Figure 1A) volume changes appeared on the "Y" axis and the mouth-to-esophagus pressure difference on the " $\mathrm{X}$ " axis. In this instance the respiratory cycles appeared as loops. At the instants of flow reversal at the "top" and "bottom" of the loops the flow-resistive component of pressure was zero and only the elastic component remained. The slope of a line

\footnotetext{
2 Sanborn Company, Cambridge, Massachusetts.
} 
drawn through the volume extremes of a loop therefore expressed the ratio of volume change to elastic pressure change. Expressed in liters per $\mathrm{cm}$. of water this slope defined the compliance of the lungs for that particular breath. (The inverse of compliance is elastance).

A second pattern was used to measure the flow-resistance of the lungs (Figure 1B). In this instance the rate of air flow appeared on the " $Y$ " axis and the flowresistive component on the " $\mathrm{X}$ " axis. The latter quantity was obtained by first registering the total mouth-toesophagus pressure difference on the " $\mathrm{X}$ " axis as before. Again a loop was produced with each respiratory cycle. By then applying the volume variation to the " $\mathrm{X}$ " axis so as to be subtracted electrically from the total pressure variation, the loop could be caused to "close" at the " $\mathrm{X}$ " axis. In effect, this subtracted from the total pressure variation just the elastic component, and the remainder represented the flow-resistive component. ${ }^{3}$ The pattern then produced showed the relationship between the rate of flow and the pressure producing this flow at every instant during the respiratory cycle. The flow-resistance at any instant was expressed by the ratio of pressure to
A

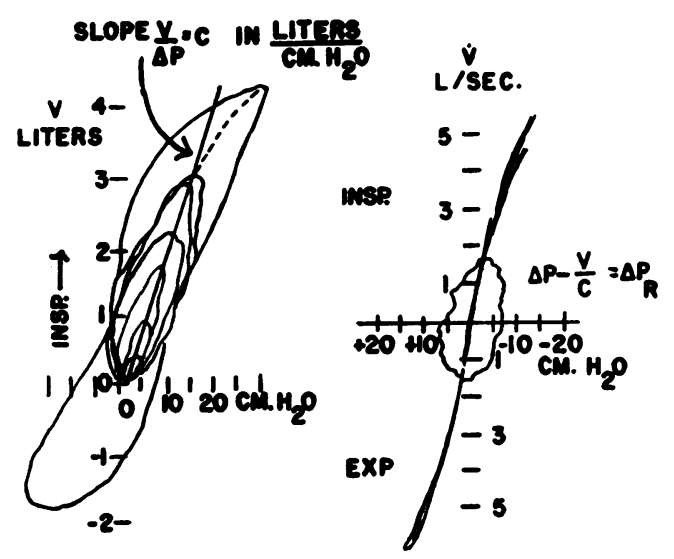

Fig. 1. Examples of the Two Types of Patterns ObTAINED WITH THE OSCILLOSCOPE

For a description of these patterns see the text.

8 This procedure is based on the following relationship. The total pressure is the algebraic sum of its elastic and flow resistance components. In the range of the resting tidal volume the elastic component is linearly related to change in volume (1-3). Therefore the change in volume multiplied by the proper constant equals the elastic component. The volume gain control on the " $\mathrm{X}$ " axis represents this constant. By adjusting this control various constants can be selected. The proper constant is obtained when the loop is closed. This follows from the fact that the flow-resistive pressure must be zero when flow is zero. Thus, as the flow passes through zero, it must intersect the " $X$ " axis at the same point both at end inspiration and end expiration, closing the loop at the " $\mathrm{X}$ " axis. simultaneous flow rate in $\mathrm{cm}$. of water per liter per second.

Tracings of the oscilloscope patterns were obtained in pencil by means of an indirect viewer.4 In instances where the "fatness" of the pressure-volume loops made accurate measurement of the points of minimum and maximum volume impossible, measurements were made directly from the Poly-Viso tracings obtained at high paper speeds (50 mm. per second).

\section{Experimental procedure}

Subjects were studied in the sitting position. The balloon was passed to the lower third of the esophagus. A nose clip was applied and the subject was instructed to breathe with the lips held firmly around the mouthpiece $(2.5 \mathrm{~cm}$. internal diameter).

Tracings were obtained during quiet breathing and during a variety of specific maneuvers which will be described with the results.

Tables I and II present pertinent clinical findings and the results of a number of conventional pulmonary function studies for the group of patients. Table III describes the normal group. The age range of the ten normal men was from 32 to 57 ; the average age was ten years less than that of the patient group.

Clinically, the patients represented a fairly homogeneous group. Their ages ranged from 29 to 69 years and all were men. Six patients had either a long history of bronchial asthma or repeated pulmonary infections; bronchiectasis was present in one. Dyspnea was always the chief complaint; in about one-half it was present at rest, and the other patients were short of breath on slightest exertion. Physical examination, chest roentgenograms and fluoroscopic observation invariably showed evidence of severe pulmonary emphysema. None of the patients was considered to have cor pulmonale, and none had an enlarged liver, ascites, or edema. The results of the conventional pulmonary function studies were consistent with the diagnoses of severe pulmonary emphysema in every instance.

\section{RESULTS}

\section{Quiet breathing}

Figure 2 shows a typical tracing of the mouthto-esophagus pressure difference, rate of flow, and volume change during quiet breathing in a patient with emphysema. A similar tracing from a normal subject is shown for comparison.

Although the tidal volumes and breathing frequencies in the two instances are nearly the same, the breathing patterns are strikingly different. Prolonged expirations and obliteration of coarse irregularities in the flow patterns were common

\footnotetext{
4 Oscillotracer, Robert A. Waters, Inc., Waltham, Massachusetts.
} 

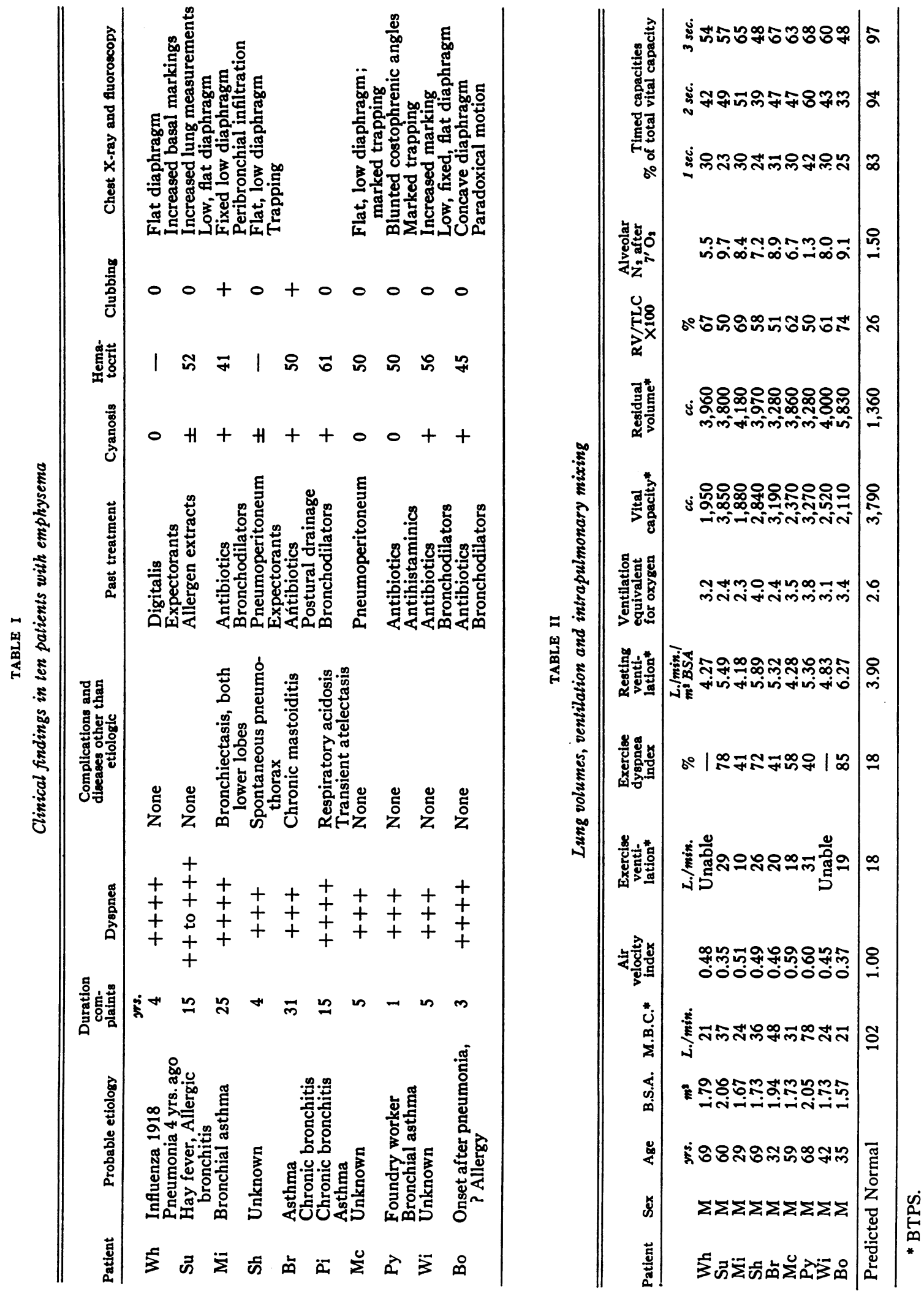
TABLE III

Age, height, and body surface area of ten normals

\begin{tabular}{lccc}
\hline \hline Name & Age & Ht. & B.S.A. \\
\hline & yrs. & $c m$. & $m^{2}$ \\
Le & 32 & 173 & 1.79 \\
Co & 54 & 162 & 1.44 \\
Mo & 55 & 163 & 1.63 \\
Af & 33 & 162 & 1.66 \\
Go & 34 & 183 & 2.04 \\
Wh & 38 & 187 & 2.12 \\
Ca & 57 & 179 & 1.89 \\
Fa & 40 & 166 & 1.74 \\
Me & 32 & 187 & 2.06 \\
Fe & 33 & 189 & 2.12 \\
\hline
\end{tabular}

to all of the patients studied. The relatively large amplitude of the pressure variation was also characteristic.

Figure 3A shows the volume-pressure tracings for the cycles shown in Figure 2. The compliance of the lungs is about the same in the two cases. (The zero flow pressure at end-inspiration was obtained from the tracings, Figure 2.) The "fatness" of the loop is much greater in the patient with emphysema. This is due to the increased resistance and is shown more clearly in Figure $3 \mathrm{~B}$ in which the flow-resistive pressure difference is on the abscissa and the rate of flow on the ordinate. In this graph inspiration and expiration both start and end at the origin. During inspiration the relationship of flow to pressure is the same for the in- creasing and decreasing phases of flow rate resulting in single curved lines. This is also the case during expiration in the normal, but the patient's expiration describes a loop. As stated above, pulmonary flow-resistance may be expressed as the pressure necessary to produce a given rate of flow. Early in expiration, the patient accomplishes a flow of 0.2 liters per second at the cost of a little more than $2 \mathrm{~cm}$. of water pressure. Later, the same flow costs more than three times this pressure. This increase in expiratory resistance is plotted against time (Figure $3 \mathrm{C}$ ). Here ratios of resistive pressure to flow are plotted at intervals during the respiratory cycle. At all times the pulmonary flow resistance of the patient is much greater than normal, and during expiration it becomes thirty times as great.

A summary of results for all of the patients and normal subjects during quiet breathing is shown in Table IV. The compliance values in all cases are the mean of at least five separate measurements. The resistance values represent the highest values measured during inspiration and expiration, corresponding to the "peaks" in Figure 3C.

The compliances of four of the patients are below the normal range, while only one lies above. The average compliance in the patients is somewhat less than in the normal individuals, suggesting that the patients' lungs tended to be somewhat

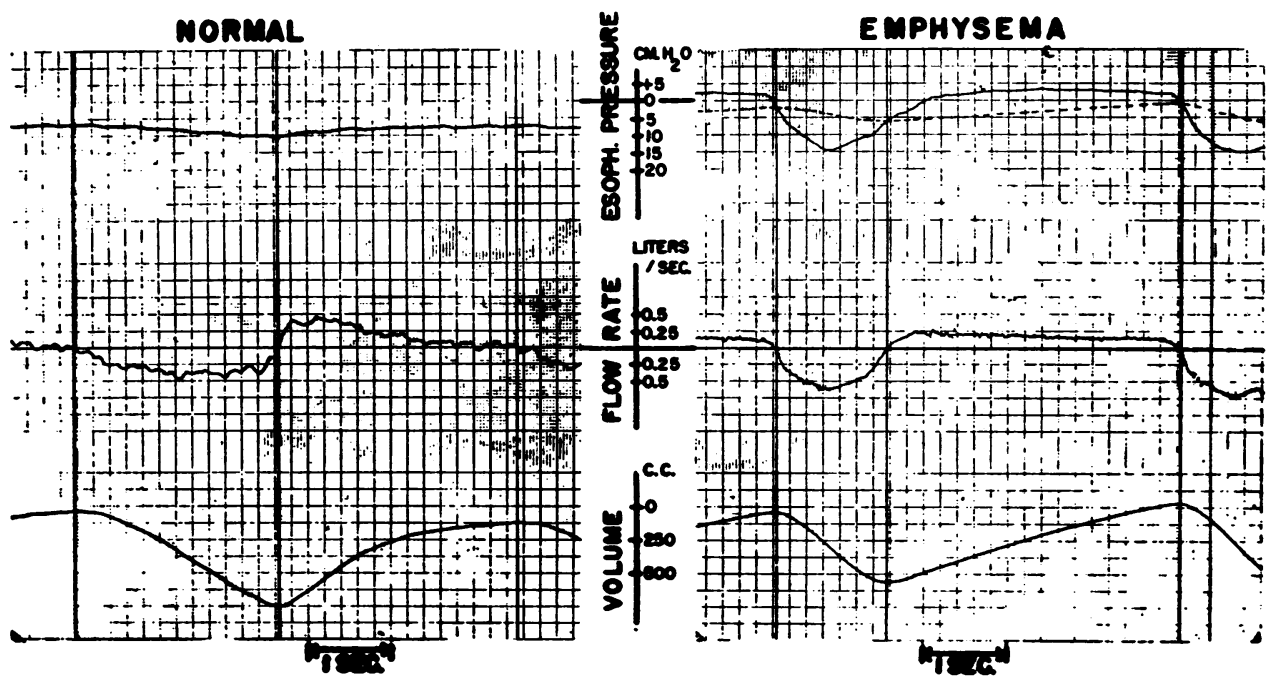

Fig. 2. Typical Tracings During Quiet Breathing in a Normal Subject and a Patient with Pulmonary Emphysema

The dotted line on the pressure tracing on the right represents the calculated elastic component of pressure change. 


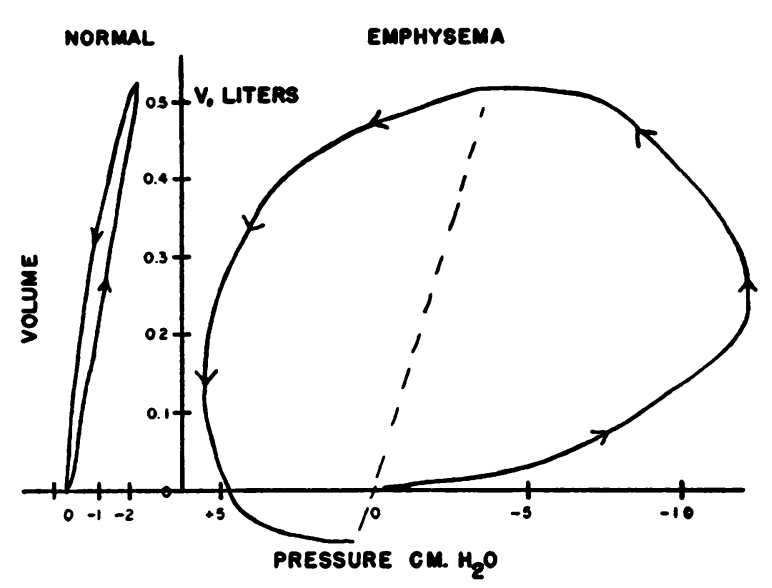

A

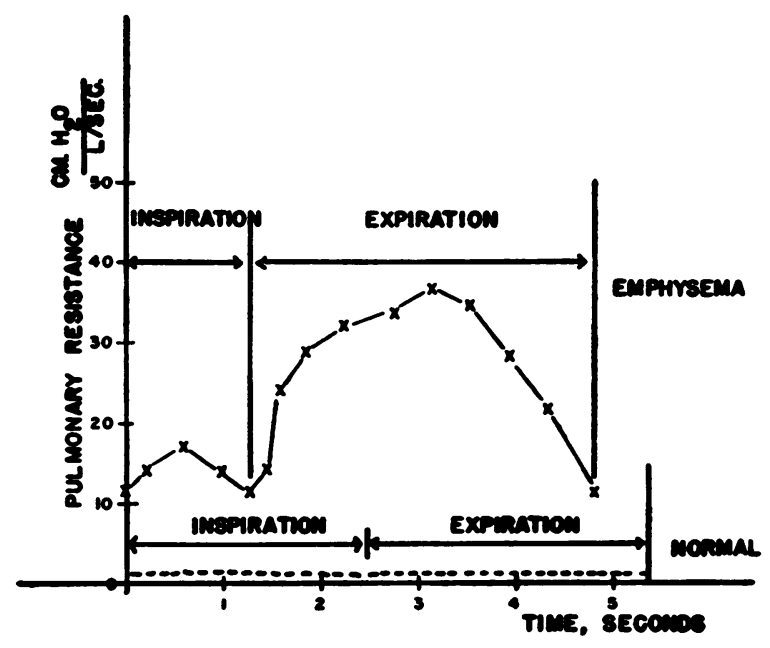

C

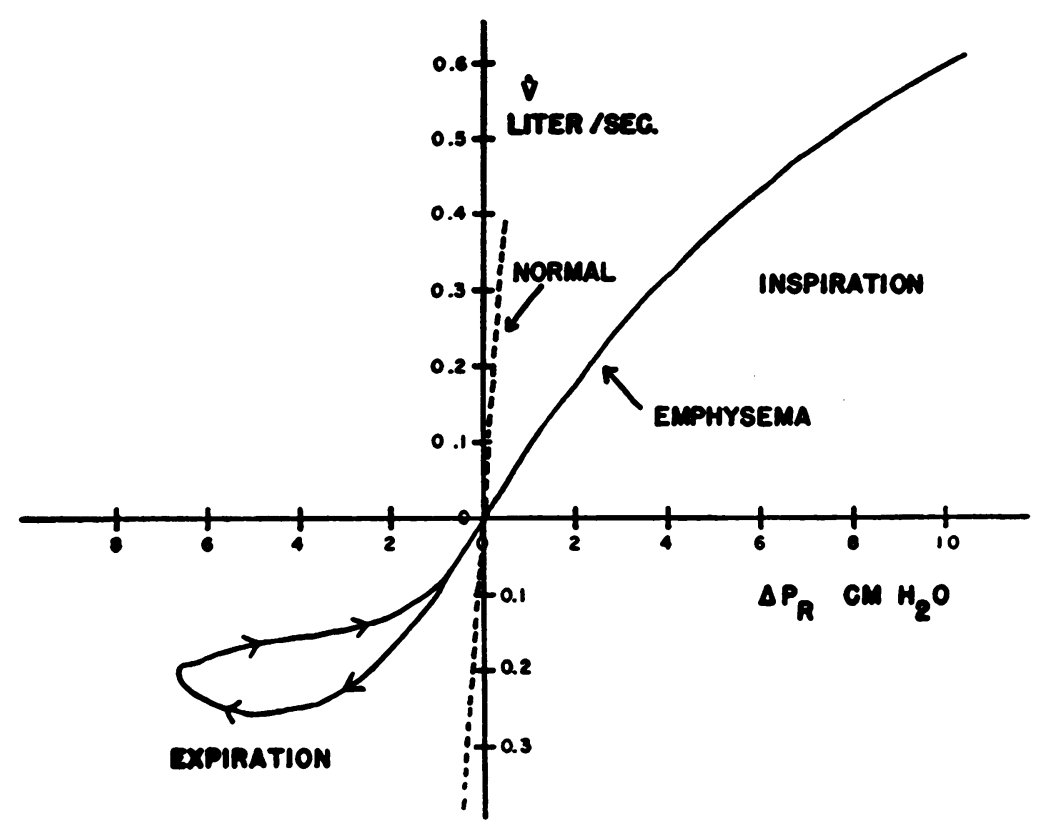

B

Fig. 3. Three Representations of the Data Shown in Figure 2. A. Pressure-Volume Graphs from Which Pulmonary Compliance May be Measured. B. Resistive Pressure-Flow Graphs from Which Pulmonary Flow Resistance May Be Measured. C. Graphs of Pulmonary Flow Resistance Changes During the ReSPIRATORY CYCLE

less compliant than normal during quiet breathing.

The differences in flow resistances between the groups are more marked. The patients have increased inspiratory flow resistance. No consistent difference in inspiratory and expiratory resistance was found in the normals. In contrast, the greatest expiratory resistance was invariably larger than the greatest inspiratory resistance in the patients.

\section{Pulmonary resistance during voluntary hyper- ventilation}

Greater differences between the two groups were brought out during hyperventilation. Fig- 
ure 4A shows typical graphs of resistances for a normal volunteer (dotted line) and a patient (solid line) during a maximally rapid inspiration followed by a maximally rapid expiration. For comparison, the entire area covered by Figure 3B showing the resistance during quiet breathing is indicated as a rectangle around the origin. The normal subject can produce far greater rates of flow. During a maximally rapid expiration the normal tracing has the same general configuration as the patient's.

Figure 4B shows flow resistance plotted against time (compare with Figure $3 \mathrm{C}$ ). Both the normal subject and the patient have marked increases

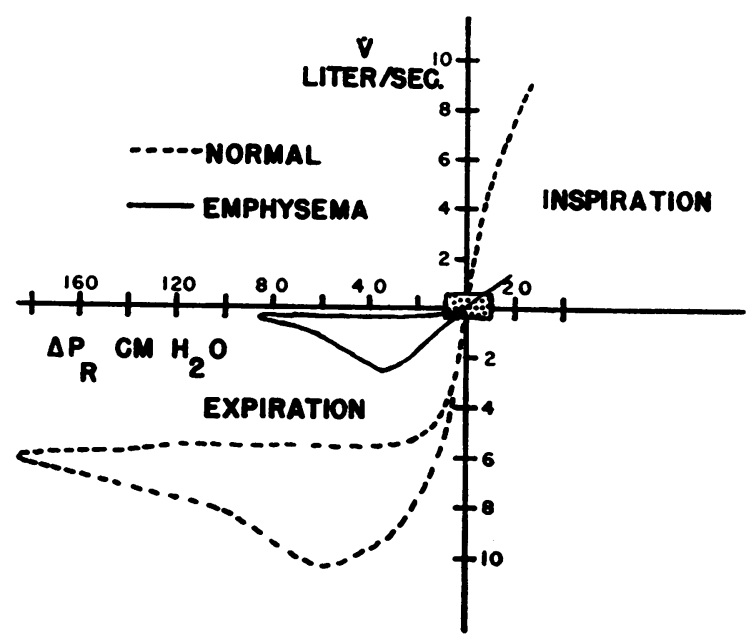

$\mathbf{A}$
TABLE IV

Pulmonary compliance and resistance during quiet breathing

\begin{tabular}{|c|c|c|c|c|}
\hline Patients & $\begin{array}{l}\text { Com- } \\
\text { pliance }\end{array}$ & $\begin{array}{c}\text { (1) } \\
\text { Greatest } \\
\text { insp. res. }\end{array}$ & $\begin{array}{c}\text { (2) } \\
\text { Greatest } \\
\text { exp. res. }\end{array}$ & $\frac{(2)}{(1)}$ \\
\hline & L. $/ \mathrm{cm} . \mathrm{H} \mathrm{H}_{9} \mathrm{O}$ & cm. $H_{2} O / L . / s e c$. & $\mathrm{cm} . \mathrm{H}_{2} \mathrm{O} / \mathrm{L} . / \mathrm{sec}$. & \\
\hline Wh & 0.14 & $5.2(0.8)^{*}$ & $14.5(0.4)^{*}$ & 2.8 \\
\hline $\mathrm{Su}$ & 0.21 & $6.4(1.0)$ & $20.0(0.5)$ & 3. \\
\hline $\mathrm{Mi}$ & 0.07 & $19.0(0.7)$ & $35.0(0.3)$ & 1. \\
\hline Sh & 0.26 & $6.9(1.0)$ & $11.5(0.6)$ & 1. \\
\hline $\mathrm{Br}$ & 0.17 & $13.0(0.6)$ & $23.0(0.8)$ & 1. \\
\hline $\mathrm{Pi}$ & 0.44 & $4.5(1.0)$ & $21.0(0.3)$ & 4. \\
\hline Mc & 0.09 & $16.5(0.6)$ & $29.0(0.3)$ & 1. \\
\hline $\mathrm{Py}$ & 0.10 & $8.9(1.0)$ & $12.0(0.6)$ & 1. \\
\hline$W i$ & 0.09 & $26.0(0.7)$ & $47.0(0.2)$ & 1.8 \\
\hline Bo & 0.17 & $10.5(0.7)$ & $34.0(0.3)$ & 3.2 \\
\hline Mean & 0.17 & 11.7 & 24.7 & 2.4 \\
\hline
\end{tabular}

\begin{tabular}{|c|c|c|c|}
\hline Normals & & & \\
\hline $\begin{array}{l}\mathrm{Le} \\
\mathrm{Co} \\
\mathrm{Mo} \\
\mathrm{Af} \\
\mathrm{Go} \\
\mathrm{Wh} \\
\mathrm{Ca} \\
\mathrm{Fa} \\
\mathrm{Me} \\
\mathrm{Fe}\end{array}$ & $\begin{array}{l}0.15 \\
0.22 \\
0.15 \\
0.20 \\
0.26 \\
0.30 \\
0.16 \\
0.13 \\
0.29 \\
0.30\end{array}$ & $\begin{array}{l}2.3 \\
1.9 \\
2.9 \\
1.2 \\
2.8 \\
1.1 \\
2.4 \\
1.7 \\
1.2 \\
1.2\end{array}$ & $\begin{array}{l}\text { Greatest expiratory resistance } \\
\text { did not differ significantly } \\
\text { from greatest inspiratory re- } \\
\text { sistance in the normal group. }\end{array}$ \\
\hline Mean & 0.22 & 1.9 & \\
\hline
\end{tabular}

* Rates of flow in L. per sec. at which resistance was greatest.

of pulmonary flow resistance during expiration. This is in contrast to quiet respiration when only the patient showed this. The tremendous increase in expiratory resistance in the patient may be appreciated by considering the observed flows

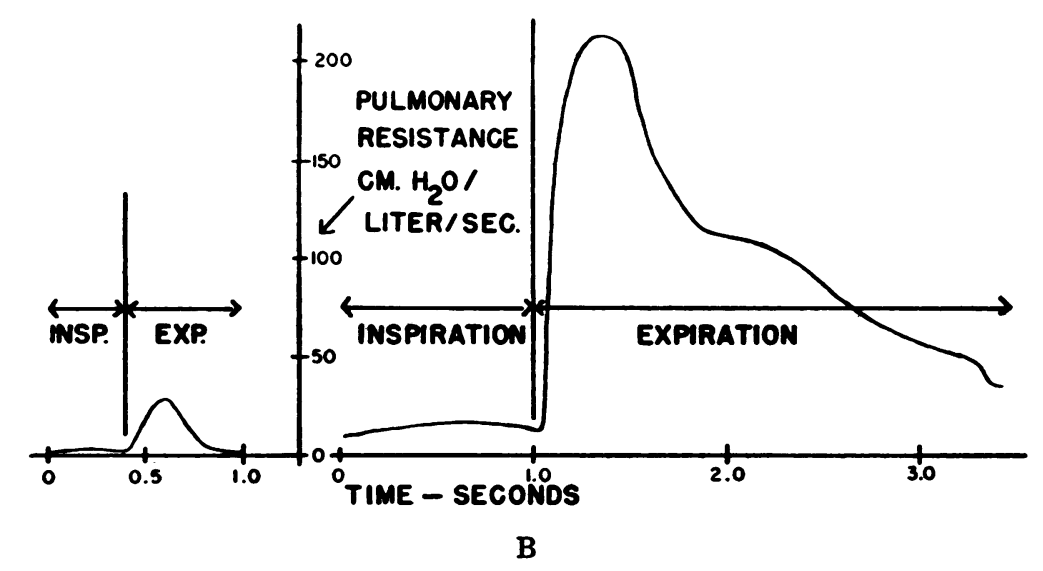

Fig. 4. A. Resistive Pressure-Flow Graphs During Maxmally Rapid Inspirations and Expirations for a Normal (Dotted Line) aNd a Patient (Solid Line). B. Flow Resistance Changes During the Course of Maximally Rapid Inspirations and Expirations for the ExAMPLes Shown IN 4A. Left, normal; right, emphysema. 
TABLE V

Pulmonary resistance during maximally rapid expirations

\begin{tabular}{|c|c|}
\hline Patient & Cm. $\mathrm{H}_{2} \mathrm{O} / \mathrm{L} . / \mathrm{sec}$. \\
\hline $\begin{array}{l}\text { Wh } \\
\text { Su } \\
\text { Sh } \\
\mathrm{Pi} \\
\mathrm{Mc} \\
\mathrm{Py} \\
\mathrm{Wi} \\
\mathrm{Bo}\end{array}$ & $\begin{array}{r}98 \\
139 \\
300 \\
122 \\
210 \\
330 \\
300 \\
480\end{array}$ \\
\hline & $\overline{247}$ \\
\hline
\end{tabular}

and pressures at the time of maximal resistance. While applying a pressure difference of more than $80 \mathrm{~cm}$. of water to his lungs and airway the rate of flow was only 0.5 liter or of the order of magnitude encountered during normal resting expiration where the resistive pressure would be less than $1 \mathrm{~cm}$. of water in normal individuals.

Table $\mathrm{V}$ shows the peak pulmonary flow-resistance values during maximally rapid expirations in eight patients. The rapid expirations were produced following maximally deep inspirations. The average increase from the resting values was ten-fold. . Pulmonary resistance during maximally rapid inspirations was approximately the same as during quiet breathing. In the normals, expiratory pulmonary resistance increased to values ranging from 10 to $30 \mathrm{~cm}$. of water per liter per second. This represented a marked increase from resting levels, but the absolute increase was approximately one-tenth that seen in the patients.

\section{Pulmonary compliance at different breathing rates}

Alteration in the breathing rate produced marked changes in the apparent elastic behavior of the patient's lungs. This was first noted during the following procedure: the patients produced tidal volumes of from 0.2 to 1.0 liter, first as slowly as possible and then with increasing frequency up to maximally rapid rates. At high breathing frequencies the rate of change of intraesophageal pressure is high at the extremes of the tidal volume, and a valid measurement of the pressures at the instants of zero air flow is possible only if the pressure and flow recording systems are capable of reproducing precisely the timing and magnitude of the pressure and flow events. Although the response characteristics of the two systems were sufficient to reproduce accurately sine waves of flow and pressure change at frequencies far greater than the peak breathing frequency observed, an additional check was made on an artificial system in which the wave forms encountered in rapid breathing could be more accurately reproduced. A flow resistance, consisting of a small pneumotachograph screen was placed in the mouthpiece assembly. The pressure drop across this resistance was measured with the esophageal balloon and the flow through the resistance with the pneumotachograph. No phase differences between pressure and flow could be demonstrated while breathing through this system at frequencies more than twice the maximal frequency observed in the patients. In the patients, pulmonary compliance progressively decreased as breathing frequency increased. In normal subjects no such alterations of compliance are observed (4). Table VI presents pulmonary compliance values obtained during quiet breathing and during maximally rapid breathing for the eight patients who performed this maneuver. It was not possible to control the patients' respiratory mid-position during these efforts and it always moved in the inspiratory direction. The changes observed were of a greater order of magnitude than could be explained by the mid-position shifts alone.

It was difficult to measure pulmonary compliance at frequencies below resting levels. In two patients it was possible to extend the measurements down to conditions of no flow by using an interrupter technique similar to that described

TABLE VI

Pulmonary compliance during quiet and rapid breathing in eight patients with pulmonary emphysema

\begin{tabular}{|c|c|c|c|c|}
\hline \multirow[b]{2}{*}{ Patient } & \multicolumn{2}{|c|}{ Quiet breathing } & \multicolumn{2}{|c|}{ Rapid breathing } \\
\hline & $\begin{array}{l}\text { Resp. } \\
\text { rate }\end{array}$ & $\begin{array}{c}\text { Pulm. } \\
\text { compliance }\end{array}$ & $\begin{array}{l}\text { Resp. } \\
\text { rate }\end{array}$ & $\begin{array}{c}\text { Pulm. } \\
\text { compliance }\end{array}$ \\
\hline \multirow{3}{*}{$\begin{array}{l}\text { Wh } \\
\mathbf{S u} \\
\mathbf{M i} \\
\mathbf{S h} \\
\mathbf{B r} \\
\mathbf{P i} \\
\mathbf{M c} \\
\mathbf{P y}\end{array}$} & & $\begin{array}{c}\text { liters/ } \\
\text { cm. } \mathrm{H} \$ \mathrm{O}\end{array}$ & & $\begin{array}{c}\text { liters/ } \\
\mathrm{cm} . \mathrm{H} / \mathrm{O}\end{array}$ \\
\hline & $\begin{array}{l}21 \\
14 \\
16 \\
23 \\
18 \\
21 \\
17 \\
16\end{array}$ & $\begin{array}{l}0.14 \\
0.21 \\
0.07 \\
0.26 \\
0.17 \\
0.44 \\
0.09 \\
0.10\end{array}$ & $\begin{array}{r}75 \\
75 \\
60 \\
100 \\
135 \\
150 \\
60 \\
45\end{array}$ & $\begin{array}{l}0.06 \\
0.09 \\
0.02 \\
0.05 \\
0.02 \\
0.03 \\
0.03 \\
0.03\end{array}$ \\
\hline & & 0.19 & & 0.04 \\
\hline
\end{tabular}




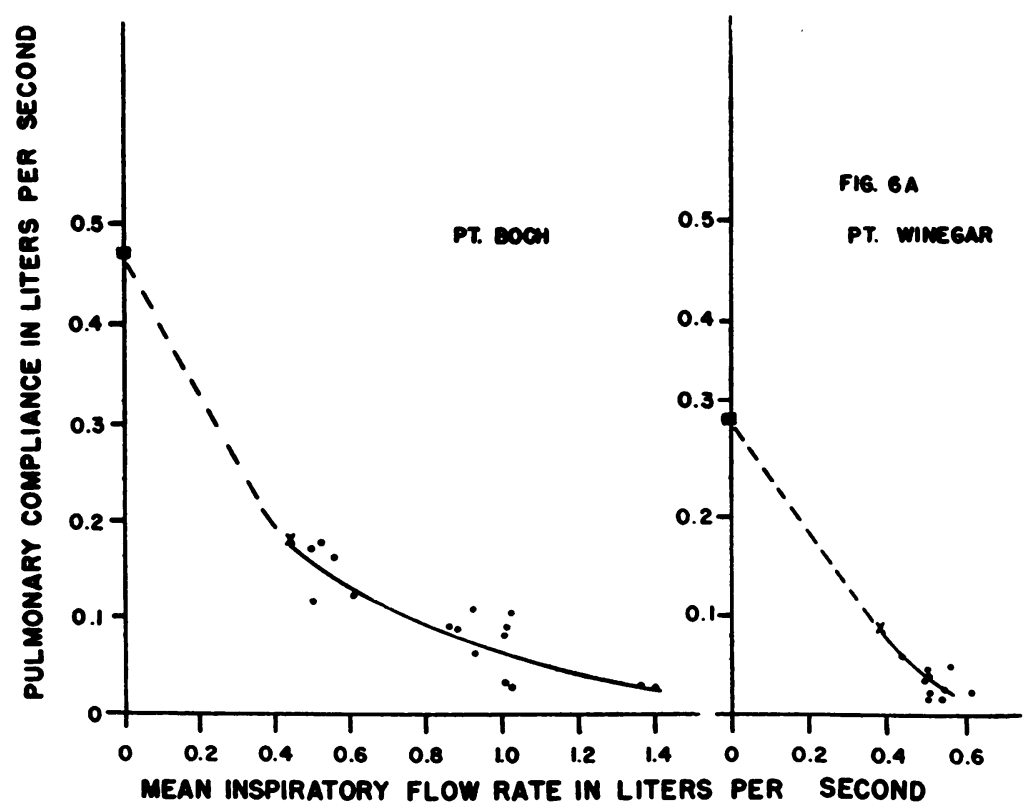

Fig. 5. Pulmonary Compliance in Two Patients

The squares on the ordinate are "static" values. The $\mathrm{X}$ 's indicate values obtained during quiet breathing and the dots, values obtained during voluntary hyperventilation.

by Stead, Fry, and Ebert (3). Mouth-to-esophageal pressure difference was measured following interruption of the air stream, in this case by means of a solenoid-driven valve in the mouthpiece assembly. The valve was triggered by a switching device attached to a spirometer from which the patient inspired. At volume increments of 0.25 liter the valve closed for pre-set time intervals. Pressure differences reached a steady level, except for the continuing oscillation synchronous with the cardiac cycle, in periods less than one second. Volume increments were plotted against esophageal pressure difference change measured at the ends of the periods of interruption. The slopes of the volume-pressure plots obtained in the region of the normal resting midposition represent "static" pulmonary compliance values if the static state may be presumed to have occurred during the two-second interruption. In Figure 5 the values obtained in this manner in the two patients are plotted as squares on the ordinates. The " $\mathrm{X}$ " marks indicate the mean of values measured during quiet respiration and the dots represent compliance values during voluntary hyperventilation. The "static" compliance is about three times greater than during quiet breath- ing and some twelve times greater than during hyperventilation.

Although "static" compliance measurements were made on only two of the patients, other evidence obtained in six patients suggests that, under conditions approaching the static stage, pulmonary compliance was increased from quiet breathing levels. During vital capacity determinations the patients were instructed to maintain the maximal inspiratory position with an open glottis for a period of several seconds before expiring maximally. Flow was near zero in these instances and esophageal pressure approached "static" conditions. In only one patient was the intra-esophageal pressure as low as $-20 \mathrm{~cm}$. of water. In the remaining patients the pressures ranged from -7 to -11 $\mathrm{cm}$. of water. In five normal individuals the pressure ranged from -25 to $-33 \mathrm{~cm}$. of water. At the instant of maximal inspiration the patients had somewhat smaller gas volumes within their lungs than the normals; total capacities in these six patients ranged from 5.2 to 7.9 liters with a mean of 6.6 while in the five normals they ranged from 7.0 to 9.0 with a mean of 8.3. Taking the ratio of the total lung capacity to the intra-esophageal pressure at the maximal inspiratory level as a rough 
measure of the over-all pulmonary compliance, the patients with one exception had greater values than the normals. (Patient range: 0.3 to 1.1 liters per $\mathrm{cm}$. of water with a mean of 0.5 . Normal range 0.26 to 0.36 with a mean of 0.3 ).

\section{DISCUSSION}

\section{The flow-resistive behavior of emphysematous lungs}

Quantitative evidence of increased expiratory flow resistance in emphysema was first obtained in one patient by Neergaard and Wirz (5). Dayman (6), and more recently Fry, Ebert, Stead, and Brown (7) have confirmed this in all of their patients. The same observation has been made in a patient with bronchial asthma (4) and in normal individuals under three specific circumstances: 1) During maximally rapid expirations $(1,7) ; 2$ ) during quiet breathing accomplished voluntarily well down in the expiratory reserve range $(1,7)$; and 3 ) during quiet breathing at a normal midposition following the inhalation of a histamine aerosol, which produced a two- to three-fold increase in respiratory flow resistance (4).

All of these observations are consistent with the "check-valve" mechanism explored in detail by Dayman. Since this mechanism may play a role in the elastic behavior of emphysematous lungs it is discussed here.

During the respiratory cycle the intrathoracic airways are subjected to alterations in pressure difference across their walls. Because the pressure on the outside of the airways more closely approximates intrapleural pressure than the pressure within the airways-which is certainly true for the extrapulmonary portions, the trachea and main bronchi-the pressure difference tends to keep the respiratory tract expanded during quiet respiration. During rapid forced expiration, however, this pressure difference is reversed, and the respiratory tract caliber reduced, thereby increasing pulmonary flow resistance. Furthermore, this sequence of events would be "self-augmenting" since increased velocity of flow would further increase the unfavorable transmural pressure difference. Conversely, during inspiration the events should be "self-limiting." Here the transmural pressure difference is reversed, tending to increase the cross-section which would lead to reduction in air-flow resistance and air-stream kinetic energy and thus reduce the transmural pressure difference. This conclusion is consistent with the observation that no equivalent reductions in pulmonary frictional resistance are observed during rapid inspiration.

Direct observations of the respiratory tract by bronchoscopy and bronchography support this hypothesis. Di Rienzo (8) has demonstrated with bronchograms tracheobronchial collapse during the rapid expiratory phase of cough efforts. Furthermore, invagination of the membranous portion of the trachea and bronchi during rapid expiratory efforts is commonly seen during bronchoscopy.

A number of possible structural changes could result in abnormal compression of the respiratory tract during expiration. Reduction in cross-section of the smaller branches of the respiratory tree could have such a result. The resulting increase in air-flow resistance would be associated with greater transmural pressure fluctuations in the intrathoracic portions of the airway "mouthward" from the points of constriction. Increased tissue frictional resistance could give a similar result.

In addition to mechanisms producing increased transmural pressure fluctuations, two additional possible predisposing factors should be mentioned. The structural integrity of the airway walls per se, could play a role to the extent that weakening of the walls would reduce the pressure necessary to cause collapse. Furthermore, as Fry, Ebert, Stead, and Brown have demonstrated (7), the elasticity of the lung plays a protective role in preventing collapse of the airways during expiration.

Of these factors only the last is clearly evident in emphysema, but any or all may play a role. Finally, although expiratory increases in resistance may be more marked in emphysema than in other conditions, they are not limited to emphysema.

\section{The elastic behavior of emphysematous lungs}

Christie (9) first noted that the "distensibility of the lungs" in emphysema appeared to be greater when measured from tracings of volume and intrapleural pressure during vital capacity maneuvers than from similar tracings during quiet breath- 
ing. Commenting on these findings, McIlroy and Christie (10) said: "The maximum inspirations were made slowly at a rate of approximately five respirations per minute as compared with the 15 to 20 per minute of the patient's normal breathing, and, in fact, these results show that during life the distensibility of the lungs in emphysema depends largely on the respiratory rate." Although "distensibility" in this instance undoubtedly included flow-resistance along with elastic behavior, it is probable that they were describing the same phenomenon here reported.

In attempting to explain the apparent decrease in compliance of emphysematous lungs during increased breathing rates, the influence of the distribution of elastic and flow-resistive properties within the lung on their over-all mechanical behavior has been considered.

The results of an animal experiment are helpful in introducing these considerations. The compliance of a dog's lung was measured at different breathing frequencies produced by a Starling pump connected to the tracheal cannula. Since the chest had been opened, the transpulmonary pressure could be measured by recording the tracheal pressure with reference to the atmosphere. Over a range of frequency from 5 to 60 cycles per minute the compliance remained constant. A catheter with a small balloon around the tip was then passed into the left main bronchus and inflated so as to block completely ventilation of the left lung. The compliance of the right lung was then measured. It was slightly more than one-half as compliant as the two lungs together, as would be expected from the relative size of the two lungs. Again the compliance did not change with frequency.

Next the balloon was slightly deflated permitting some ventilation of the left lung, but the resistance to flow was greater than normal. In this instance when volume changes were produced slowly the two lungs had nearly equal tidal excursions. The compliance was the same as that measured for the two lungs before the catheter was passed. As the rate was increased it was observed that the partially obstructed lung participated less in the total volume change and its movement appeared to lag behind that of the right lung. The compliance measured in this instance had decreased. With further increase in rate, compli- ance fell to levels nearly the same as recorded for the right lung alone. At these rapid rates the volume change of the left lung was barely noticeable; almost all of the flow was into and out of the right lung.

This experiment illustrates in gross fashion the influence of an abnormal distribution of resistance on the mechanical behavior of lungs. At low frequencies, when the rates of flow were low, the resistance discrepancy had little effect on the distribution of flow to the two lungs. In this instance the compliance of the lungs determined the distribution of volume change, and the over-all system compliance was the sum of the individual lung compliances. As the cycling frequency of the pump was increased the differences in flow resistance to the two lungs had a progressively greater effect on the distribution of flow to the two lungs. Functionally, the apparent reduction in compliance reflected this change.

The concepts illustrated by this experiment can be extended to points further along the respiratory tree. At every point of branching the distribution of flow between the two branches depends on the elastic and flow-resistive properties of the two pathways. When the rates of flow are low this distribution depends chiefly on the elastic properties of the pathways. As the rates of flow increase, the distribution depends increasingly, and at high rates of flow predominantly on the flow resistance of the two pathways.

The only circumstance under which the overall compliance of the lungs will remain unchanged with changes in breathing rate is one in which the distribution of flow within the lungs remains the same as the breathing frequency changes. It is apparent that flow-resistive and elastic properties would have to be distributed in a special way to permit this. In attempting to understand the nature of such a distribution, techniques developed in the field of electrical engineering are useful. Certain electrical networks are analogous to the lungs' mechanical networks, and the theory that has been developed to account for their behavior can be applied to the lungs! It is not within the scope of this report to present such an analysis. ${ }^{5}$ The conclusions which may be reached can be expressed simply:

\footnotetext{
5 Details of this analysis will appear in Reference 4.
} 
Considering any pathway in the lung, the product of the flow resistance and compliance of the pathway has the unit of time:

$$
\begin{aligned}
& \text { Compliance } \times \text { Resistance }=\text { Time } \\
& \frac{\text { Volume }}{\text { Pressure }} \times \frac{\text { Pressure }}{\text { Volume } / \text { Time }}=\text { Time }
\end{aligned}
$$

This product is called the time-constant of the pathway. It can be shown theoretically and demonstrated experimentally, that if the time-constants of all the separate pathways are equal the distribution of flow within a system such as the lungs is independent of frequency. Only under this circumstance is the measured over-all compliance also independent of frequency. Any time-constant inequality, whether the result of changes in local elastic properties, flow resistive properties, or a combination of both, will result in changes in the distribution of flow with changes in frequency, and reduction in the over-all compliance as frequency is increased.

Certain corollaries of this analysis may be mentioned. The finding of unchanged compliance with changes in breathing rates in normal subjects suggests that the distribution of ventilation in normal lungs also remains the same over a wide range of frequencies. By this, equality of ventilation to all parts of the lung is not implied. It merely means that the distribution, such as it is, does not change. In contrast, the marked reduction in compliance with increasing frequency observed in emphysema implies that even during quiet breathing a considerable part of the uneven ventilation, known to exist in emphysema, must relate to dynamic factors. In all probability these abnormalities are emphasized with increasing ventilation during exercise.

It is not possible to say whether local changes in elastic or resistive properties, or both (including the check valve mechanism) are responsible for the changes in compliance with changing frequency in emphysema. It is of interest to speculate about the possible influence of this phenomenon on the pathogenesis of the disease. If the distribution of increases in air-flow resistance is non-uniform within the lungs, those parts of the lungs with the lowest resistance would become abnormally stressed, since they would undergo greater than normal volume change. The high resistance parts of the lungs would tend to be protected. Large and relatively rapid changes in volume, as during the inspiratory phase of cough, would tend to accentuate this differential stress within the lungs. Thus, it is possible that this phenomenon may play a role in the process of parenchymal destruction and lead from asthma and chronic bronchitis to emphysema.

The differential diagnosis between pulmonary emphysema and bronchial asthma is important since the former implies irreversible structural damage while the latter does not. From the standpoint of pulmonary mechanics the two conditions have much in common. In both conditions increases in pulmonary flow resistance are greatest during expiration and pulmonary compliance falls with increased rates of breathing. Theoretically certain mechanical differences should be measurable. The static compliance of the lungs of the patient with emphysema, as indicated roughly by the extent to which he can lower his intra-esophageal pressure during a slow maximally deep inspiration, should be higher than normal. (The presence of cor pulmonale in emphysema might negate this distinction.) Furthermore, the asthmatic patient studied on his "best day" or following the administration of bronchodilators might be expected to show little change in compliance with frequency and near-normal pulmonary flow resistance, while the patient with emphysema under similar circumstances would still show marked mechanical abnormality.

\section{SUM MARY}

Measurements of the mechanical properties of the lungs in ten patients with advanced pulmonary emphysema were compared with similar measurements in ten normal subjects.

Pulmonary flow resistance was found to be increased in emphysema, more so during expiration than during inspiration. Pulmonary flow resistance during expiration was further increased by increased effort.

In the patients, pulmonary compliance was near normal levels during quiet breathing, but fell markedly as the breathing frequency was increased.

Possible local mechanisms accounting for these changes are discussed and the implications of the distribution of mechanical properties within the 
lungs on their over-all mechanical behavior considered.

\section{REFERENCES}

1. Mead, J., and Whittenberger, J. L., Physical properties of human lungs measured during spontaneous respiration. J. Applied Physiol., 1953, 5, 779.

2. Buytendijk, H. J., Oesophagusdruk en longelasticiteit. Groningen, 1949, Electrische Drukkerij I. Oppenheim, N. V.

3. Stead, W. W., Fry, D. L., and Ebert, R. V., The elastic properties of the lung in normal men and in patients with chronic pulmonary emphysema. J. Lab. \& Clin. Med., 1952, 40, 674.

4. Otis, A. B., McKerrow, B. R., Mead, J., Mcllroy, M. B., Selverstone, N. J., and Radford, E. P., Jr., Some mechanical factors in the distribution of ventilation. In preparation.
5. Neergaard, K. von, and Wirz, K., Uber eine Methode zur Messung der Lungenelastizität am lebenden Menschen, insbesondere beim Emphysem. Ztschr. f. klin. Med., 1927, 105, 35.

6. Dayman, H., Mechanics of airflow in health and in emphysema. J. Clin. Invest., 1951, 30, 1175.

7. Fry, D. L., Ebert, R. V., Stead, W. W., and Brown, C. C., The mechanics of pulmonary ventilation in normal subjects and in patients with emphysema. Am. J. Med., 1954, 16, 80.

8. Di Rienzo, S., Bronchial dynamism. Radiology, 1949, 53, 168.

9. Christie, R. V., The elastic properties of the emphysematous lung and their clinical significance. $\mathrm{J}$. Clin. Invest., 1934, 13, 295.

10. McIlroy, M. B., and Christie, R. V., A post-mortem study of the visco-elastic properties of normal lungs. Thorax, 1952, 7, 291. 